\title{
Esfinter Urinario Artificial "FlowSecureTM": Un nuevo concepto de esfinter artificial regulable y con oclusión condicional para la incontinencia urinaria de esfuerzo
}

\author{
García Montes $\mathrm{F}^{* *}$, Knight SL*, Greenwell T*, Mundy AR*, Craggs MD*. \\ *Institue of Urology and Nephrology. University College of London. \\ **Hospital Universitario Son Dureta. Palma de Mallorca. \\ Actas Urol Esp. 2007;31(7):752-758
}

\section{RESUMEN}

ESFÍNTER URINARIO ARTIFICIAL "FLOWSECURETM": UN NUEVO CONCEPTO DE ESFÍNTER ARTIFICIAL REGULABLE Y CON OCLUSIÓN CONDICIONAL PARA LA INCONTINENCIA URINARIA DE ESFUERZO

Introducción: Difundir el concepto de un nuevo esfinter urinario artificial con oclusión condicional para el tratamiento de la incontinencia urinaria de esfuerzo. El prototipo fue concebido y diseñado por los profesores Craggs $\mathrm{M}$. $\mathrm{y}$ Mundy A.R. en el Instituto de Urología y Nefrología de Londres.

Materiales y Métodos: El esfinter FlowSecure es un prototipo de una sola pieza de silicona llena de suero salino que consta de: 1) Un reservorio regulador de presión, 2) un reservorio de asistencia al estrés, 3) una bomba de control con un puerto autosellable y 4) un manguito. El reservorio regulador determina la presión de la prótesis y se puede ajustar desde 0 a $80 \mathrm{~cm} \mathrm{H}_{2} \mathrm{O}$ mediante la inyección o extracción de líquido a través del puerto autosellable (oclusión basal). El reservorio de asistencia al estrés transmite de forma reversible los aumentos de la presión intra-abdominal durante los periodos de esfuerzo (oclusión condicional).

Resultados: La técnica quirúrgica de implantación es simple, reduciendo la posibilidad de infección y errores de ensamblaje. La asociación en serie de un reservorio regulador de presión con un reservorio de asistencia al estrés permite establecer presiones de oclusión basal bajas y el aumento condicional de la misma durante los periodos de estrés, reduciendo el riesgo de atrofia y erosión. Tras la implantación, la presión es ajustable dependiendo de las necesidades clinicas del paciente.

Discusión: El esfinter FlowSecure está diseñado para corregir la incontinencia ajustando la presión de oclusión según las necesidades individuales de cada paciente, así como para reducir las complicaciones asociadas al exceso de presión de oclusión y los fallos mecánicos.

Palabras clave: Esfinter urinario artificial. Oclusión condicional.

\begin{abstract}
"FLOWSECURETM" ARTIFICIAL URINARY SPHINCTER: A NEW ADJUSTABLE ARTIFICIAL URINARY SPHINCTER CONCEPT WITH CONDITIONAL OCCLUSION FOR STRESS URINARY INCONTINENCE

Introduction: To spread de concept of a new artificial urinary sphincter with conditional occlusion for stress incontinence. The new prototype was conceived and designed in The Institute of Urology and Nephrology of London by Professor Craggs M. and Professor Mundy A.R.

Methods: The FlowSecure sphincter consists of an adjustable pressure-regulating balloon, a stress relief reservoir, a control pump and valve assembly unit with self-sealing port and a urethral cuff. The pressure regulating balloon determinates de operating pressure of the device; the pressure is adjustable in the range $0-80 \mathrm{~cm} \mathrm{H}_{2} \mathrm{O}$ and can be altered by injection or removal of normal saline through the self sealing port. The stress relief balloon transmits transient intrabdominal pressure to the cuff during periods of stress. An adjustable circular urethral cuff minimises creasing and possible stress fractures.

Results: The device is implanted as a one-piece assembly which is pre-filled with sterile saline. The surgical technique is simple and associated with little handling, reducing risk of infection and potential assembly errors. The adjustable pressure regulating balloon in association with the stress relief reservoir enables the cuff occluding pressure to be set at a low range, therefore reducing the risk for atrophy and erosion.

Discussion: The new FlowSecure urinary artificial sphincter with conditional occlusion is designed to provide good continence rates adjusting regulating pressures when needed and conceived to reduce the risk of potential complications associated with excessive occluding pressures and mechanical failures.
\end{abstract}

Keywords: Artificial urinary sphincter. Conditional occlusion. 
$\mathrm{E}$ n 1947, Foley diseñó el primer esfinter urinario artificial $^{1}$ que consistía en un anillo que se colocaba alrededor del pene y se inflaba o desinflaba por medio de una bomba de control oculta en el bolsillo del paciente. A parte del estético, el principal problema que este prototipo ocasionaba era la erección que inevitablemente se producía cuando el anillo se encontraba inflado. Para evitar este problema, Foley desarrolló una técnica quirúrgica que permitía implantar el anillo alrededor de la uretra sin incluir los cuerpos cavernosos y, por tanto, restablecer la continencia de los pacientes incontinentes sin que se produjera erección alguna.

El segundo hito importante en la historia del esfinter urinario artificial se produce en 1972, cuando Scott, Bradley y Timm introducen el modelo AS-721, un prototipo cuyos componentes quedaban totalmente implantados en el interior del cuerpo y se podía manipular desde el exterior ${ }^{2}$. De todas formas, debido a la complejidad de la prótesis, la implantación era laboriosa y con frecuencia alguno de los componentes fallaba. Durante los años sucesivos se produjeron múltiples modificaciones (AS-761, AS-742, AS-791 y AS-792) para facilitar el acto quirúrgico y reducir la frecuencia de fallos mecánicos (Fig. 1). El proceso culmina en 1983 con la aparición del esfinter AMS-800, el único disponible en el mercado durante los últimos 23 años. A pesar de los resultados satisfactorios publicados con la utilización del AMS-800, la tasa de revisiones por complicaciones supera el 30\% e incluyen desde la reaparición de incontinencia por atrofia uretral hasta retirada de la prótesis por infección, erosión o fallo mecánico ${ }^{3-6}$.

Desde 1986 los profesores Craggs y Mundy ${ }^{7,8}$ concibieron una serie de modificaciones sobre el modelo AMS-800 que incluían una bomba de control con un puerto autosellable para modificar el volumen de la prótesis sin necesidad de intervención, un manguito más adaptable a la uretra con menor probabilidad de erosión o perforación y la introducción de un reservorio adicional sensible al aumento de presión intraabdominal en un prototipo de una sola pieza para facilitar la técnica quirúrgica y disminuir la incidencia de fallos mecánicos a la vez que proteger la uretra de la excesiva presión continua que ejerce el AMS-800. El resultado fue el esfínter artificial "FlowSecure".

\section{MATERIAL Y MÉTODOS}

El esfinter urinario artificial FlowSecure (Fig. 2) es un prototipo de silicona de una sola pieza que se presenta lleno con unos $30 \mathrm{ml}$ de suero salino $0,9 \%$ y está constituido por 1 ) un reservorio que regula la presión de la prótesis y establece la presión basal de oclusión; 2) un segundo reservorio colocado en serie con el primero, denominado reservorio de asistencia al estrés, que permite la oclusión condicional del manguito de forma reversible cuando aumenta la presión intra-abdominal; 3) un manguito adaptable a la uretra y 4) una bomba de control con una válvula de resistencia, un puerto autosellable y un mecanismo accesorio de llenado rápido.

\section{El reservorio regulador de presión}

Es el componente del sistema que determina la presión de oclusión basal ejercida por el manguito.

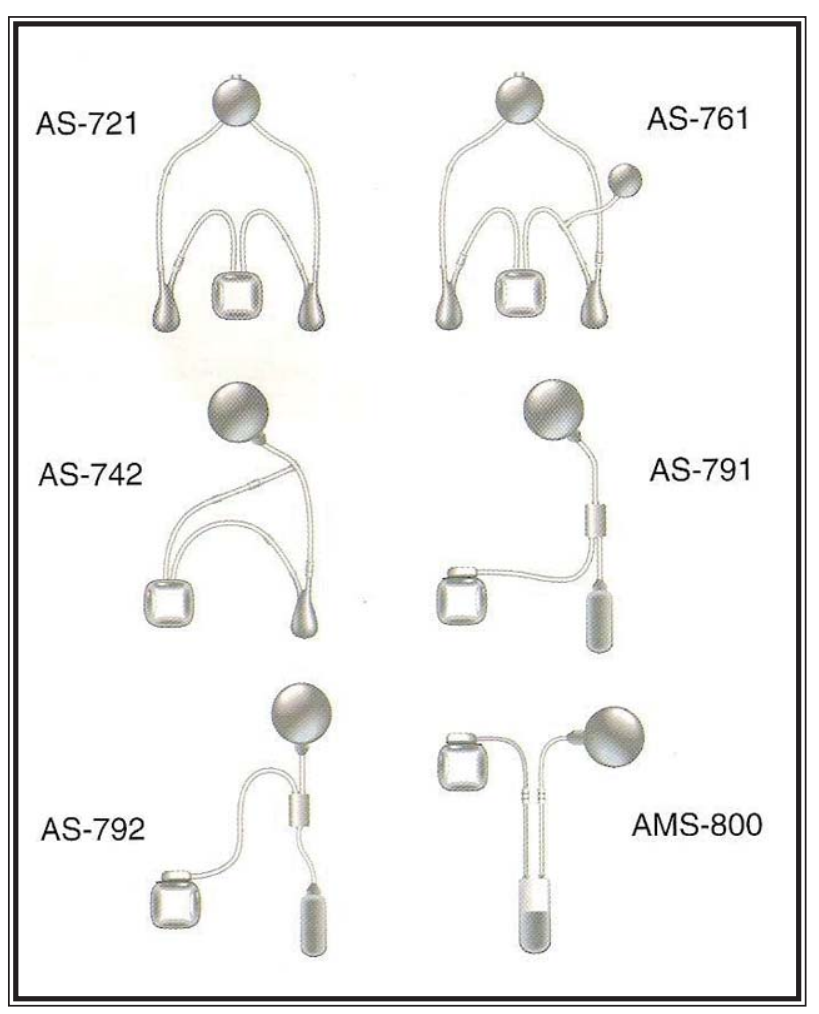

FIGURA 1. Cambios producidos en el diseño de los esfinteres urinarios artificiales desde el AS-721 en 1972 hasta el AMS-800 en 1983 para facilitar el acto quirúrgico y reducir la frecuencia de fallos mecánicos. 


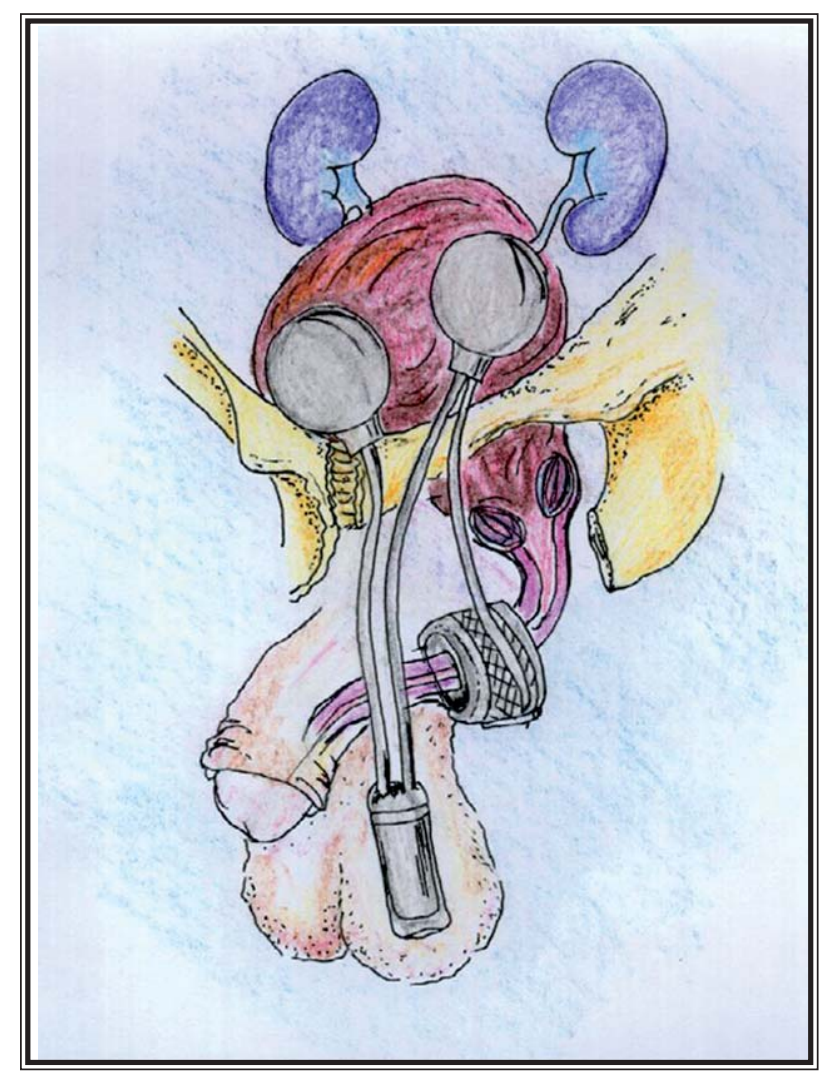

FIGURA 2. Esfinter urinario artificial "FlowSecure" ${ }^{T M}$ ". 1) Reservorio regulador de presión. Responsable de la presión de oclusión basal. 2) Reservorio de asistencia al estrés. Responsable de la oclusión condicional cuando aumenta la presión intra-abdominal durante el esfuerzo. 3) Manguito. Diseño circular y en cinturón para favorecer la adaptación a cada uretra en particular, homogeneizar la transmisión de presión y disminuir la probabilidad de aparición de grietas en la silicona. 4) Bomba de control con puerto autosellable en la base para la administración o extracción de líquido y mecanismo accesorio de llenado rápido del manguito. (Imagen del archivo personal del Profesor M. D. Craggs).

Dicha presión de oclusión es ajustable entre 0 y $80 \mathrm{~cm} . \mathrm{H}_{2} \mathrm{O}$ y puede ser modificada mediante la inyección o la extracción de volumen a través del puerto autosellable de la bomba dependiendo de las necesidades clínicas del paciente. El reservorio regulador transmite su presión al manguito a través de la bomba de control y del reservorio de asistencia al estrés (véase la Figura 2). La bomba de control presenta una válvula que permite el libre paso de líquido hacia el manguito pero impide su retorno. Tan sólo cuando el paciente comprime el bulbo de la bomba para orinar, la válvula invierte transitoriamente el paso del líquido, volviendo a recuperar la dirección normal de forma espontánea una vez se ha dejado de comprimir la bomba de control.

\section{Reservorio de asistencia al estrés}

El reservorio de asistencia al estrés es idéntico al reservorio regulador, está situado en serie con éste y se encuentra conectado directamente con el manguito, sin que el líquido pase por la bomba de control (véase la Figura 2). Transmite los cambios de presión intra-abdominal aumentando de forma inmediata la presión de oclusión uretral cuando se produce un aumento de la presión intra-abdominal y viceversa. Este mecanismo se denomina oclusión condicional y permite que la presión de oclusión aumente tan sólo cuando es necesario por el aumento de la presión intra-abdominal, protegiendo la pared uretral de la isquemia resultante de la oclusión permanente e innecesariamente elevada del manguito. La asociación en serie del reservorio regulador y del reservorio de asistencia al estrés permite establecer presiones basales de oclusión uretral bajas (reservorio regulador) y que la presión de oclusión condicional (reservorio de asistencia) aumente sólo durante el tiempo que la presión intra-abdominal se encuentra elevada. Se ha calculado que el reservorio de asistencia al estrés transmite casi el $100 \%$ de la presión intra-abdominal y tarda 200 milisegundos en transmitir el aumento de presión al manguito.

\section{La bomba de control}

La bomba de control contiene el bulbo para vaciar el manguito, un mecanismo accesorio de vaciado rápido y un puerto autosellable para la inyección o extracción de volumen de suero salino dependiendo de las necesidades clínicas. Presenta una válvula de resistencia que, en condiciones normales, sólo permite el paso de líquido desde el reservorio regulador hasta el manguito. Tan sólo cuando se comprime el bulbo de la bomba se desactiva la resistencia, permitiendo el paso de líquido en dirección contraria. Cada vez que se comprime el bulbo se transfiere líquido desde el manguito hasta el reservorio y el paciente debe continuar presionando hasta que consiga orinar con un buen flujo. Una vez vaciado el manguito, éste vuelve a llenarse automáticamente aunque existe un mecanismo accesorio que el 
paciente puede utilizar para que el manguito se vuelva a llenar más rápidamente si no desea esperar a que lo haga automáticamente.

\section{E1 manguito}

Es el componente al que se transmite la presión basal del reservorio regulador (presión basal de oclusión) así como la presión extra del reservorio de asistencia al estrés cuando se produce el aumento de la presión intra-abdominal (oclusión condicional). El manguito está diseñado de tal forma que es adaptable a la pared uretral a modo de cinturón. Presenta una cinta que permite ajustarlo a la circunferencia uretral hasta un máximo de $7 \mathrm{~cm}$. Aunque está medida podría ser utilizada en muchos de los cuellos vesicales femeninos, el manguito del Flowsecure está diseñado específicamente para la uretra masculina y aún no ha sido utilizado en mujeres. Para ajustarlo a la uretra, el manguito está provisto de dos hebillas por las que se pasa la cinta. El fabricante proporciona un adhesivo especial para la silicona, pero la falta de garantía de sellado obliga a suturar la cinta a una de las hebillas una vez que se ha adaptado a la uretra. La superficie interna del manguito es circular, por lo que la transmi-

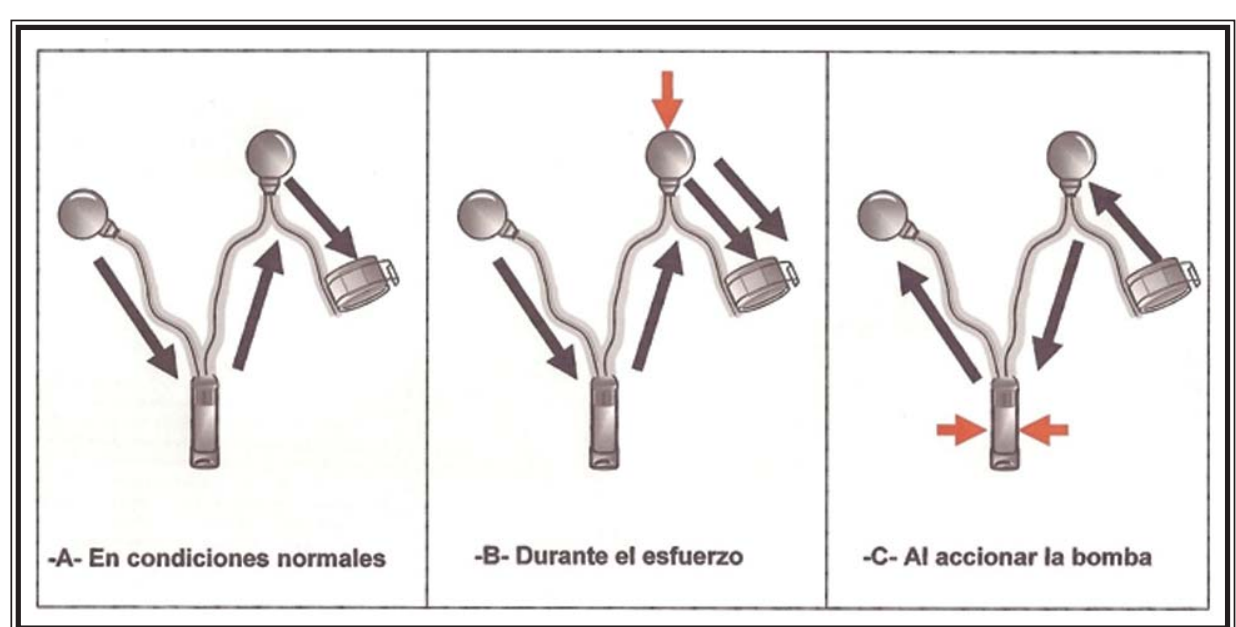

FIGURA 3. Mecánica hidráulica del modelo FlowSecure: -A- En condiciones normales el reservorio regulador transmite su presión hasta el manguito a través de la bomba de control y del reservorio de asistencia al estrés (presión de oclusión basal). -B-Durante el esfuerzo el reservorio de asistencia al estrés transmite el aumento de presión intra-abdominal directamente al manguito. Cuando cede la presión intra-abdominal también lo hace la presión del manguito (presión de oclusión condicional). -C- Cuando el paciente acciona la bomba de control el líquido del manguito pasa al reservorio regulador a través del reservorio de estrés y la propia bomba de control. El manguito se vacía y el paciente puede orinar. Pasados unos minutos la presión del reservorio regulador vuelve $a$ inflar el manguito restableciéndose la presión de oclusión basal. (Imagen modificada del archivo personal del Profesor M. D. Craggs) sión de la presión es homogénea (disminuyendo la probabilidad de atrofia y erosión) y se minimiza la creación de grietas en la silicona disminuyendo la posibilidad de perforación).

\section{Los tubos de conexión y el líquido}

El sistema de tubos de conexión está especialgislación Europea sólo prevé la duración de en el cuerpo humano durante un de días. Queda a la libre decisión de cada urólogo en particular añadir contraste al sistema a través de la bomba de control, aunque al tratarse de un modelo de una sola pieza la utilización del mismo parece poco justificada y se tendrá que tener en cuenta que la solución final debe ser iso-osmolar. Además, el funcionamiento de la prótesis se puede comprobar mediante eco-

\section{La mecánica hidráulica del sistema}

El sistema supone la máxima expresión de las leyes hidráulicas aplicadas a la urología. La prótesis sólo funciona cuando está llena de líquido, el cual pasa del reservorio regulador de presión al reservorio de estrés (a través de la bomba de control) y de éste directamente al manguito (véase la figura 3). Cuando el sistema está en funcionamiento, el paso de líquido se produce siempre desde el reservorio regulador de presión hasta el manguito, que se infla progresivamente disminuyendo su luz; de esta forma, la onda de presión hidráulica se convierte en una fuerza oclusiva. En condiciones basales el reservorio regulador ejerce una pre- 
sión constante en la uretra que no suele superar $40 \mathrm{~cm}$ de $\mathrm{H}_{2} \mathrm{O}$, lo que es suficiente para mantener la continencia en reposo sin provocar daño tisular. Cuando aumenta la presión intra-abdominal, por ejemplo al toser, ésta se transmite al reservorio de asistencia al estrés y de él al manguito en una fracción de segundo, evitando así la incontinencia de esfuerzo. En cuanto cede la presión intra-abdominal el manguito se desinfla hasta la presión basal dictada por el reservorio regulador.

Cuando el paciente desea orinar acciona el bulbo de la bomba de control repetidamente hasta que comience a orinar con buen flujo. La compresión del bulbo de la bomba de control aspira el líquido del manguito y lo transfiere al reservorio regulador. Posteriormente el manguito se vuelve a llenar de forma espontánea debido al exceso de presión generado en el reservorio regulador; la resistencia de la bomba de control enlentece el llenado del manguito, permitiendo que el paciente pueda vaciar su vejiga. Tras unos minutos el manguito se encontrará totalmente lleno a la presión basal y la continencia se restablece. En los casos en los que el tiempo de llenado espontáneo del manguito sea excesivo y el paciente gotee orina una vez completada la micción se puede manipular el mecanismo accesorio de llenado rápido, que el paciente deberá aprender a utilizar correctamente.

\section{RESULTADOS}

El resultado es un nuevo modelo de esfinter urinario artificial ajustable a las necesidades clínicas de cada paciente en particular y con oclusión condicional, diseñado para compensar de forma reversible los cambios rápidos de presión intra-abdominal a través del reservorio de asistencia al estrés, lo que permite al aumento de la presión de cierre uretral tan sólo cuando es necesario. La oclusión condicional permite, a su vez, que el reservorio regulador trabaje en un rango bajo de presiones para mantener la continencia en reposo, lo que protege la pared uretral de cambios isquémicos. El diseño del manguito permite una mejor adaptación a la pared uretral y reduce la posibilidad de formación de grietas y por tanto de perforación. El puerto autosellable de la bomba de control permite implantar el manguito a presión atmosférica cero, para posteriormente inyectar o extraer líquido del sistema siempre que sea necesario según las necesidades clínicas del paciente y sin necesidad de reintervención (Fig. 4). El mecanismo accesorio de llenado rápido del manguito permite que los pacientes puedan accionarlo si el llenado automático fuese demasiado lento como para corregir la incontinencia del periodo post-miccional. El diseño de todo el sistema en una sola pieza facilita el acto quirúrgico, que puede realizarse en unos 30 minutos, evita posible entrada de aire o partículas de sangre y grasa durante la intervención ya que no es necesario el proceso de conexión y minimiza la incidencia de fracaso mecánico. Aunque está diseñado para la uretra de varones adultos y la longitud del manguito es de $7 \mathrm{~cm}$, es posible que se pueda colocar en la mayoría de los cuellos vesicales femeninos.

\section{DISCUSIÓN}

A pesar de que el esfinter urinario artificial AMS-800 ha sido durante las dos últimas décadas una buena opción terapéutica para el trata-

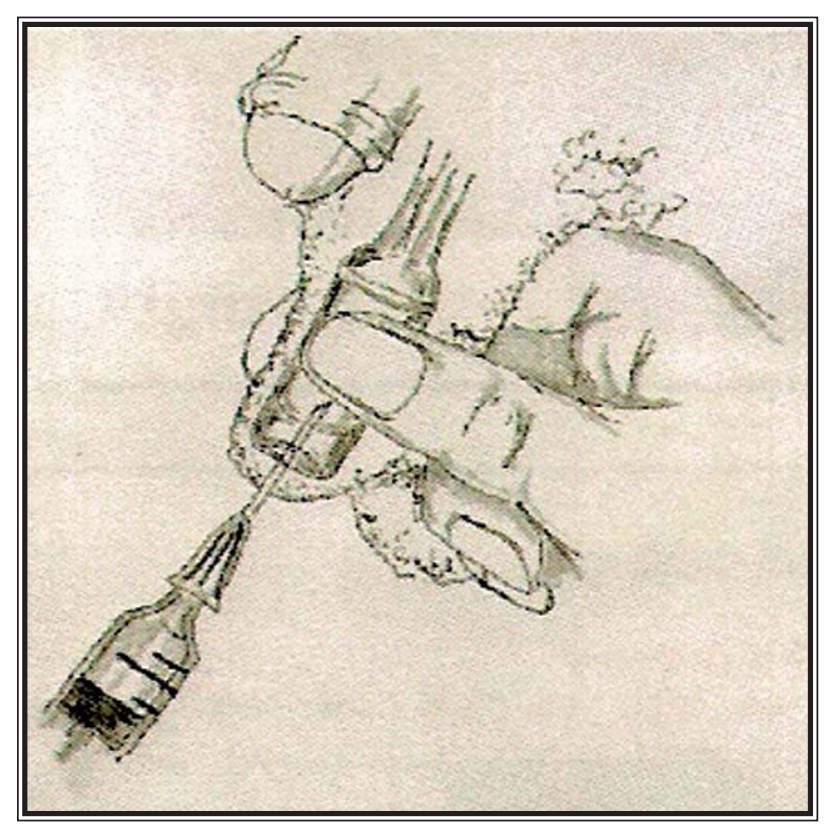

FIGURA 4. La existencia de un puerto autosellable en la base de la bomba de control permite ajustar el volumen, y por lo tanto la presión, a la que trabaja la prótesis dependiendo de las necesidades clínicas del paciente. Si persiste o reaparece la incontinencia se administra suero salino y si tiene dificultad para orinar se extrae liquido del sistema. (Imagen del archivo personal del Profesor M. D. Craggs). 
miento de la incontinencia urinaria de esfuerzo refractaria a otros tratamientos, la implantación de la prótesis no está exenta de complicaciones y la tasa de revisiones quirúrgicas supera el 30\% e incluyen desde la reaparición de incontinencia por atrofia uretral hasta retirada de la prótesis por infección, erosión o fallo mecánico ${ }^{3-6}$. Parece irónico que la complicación más frecuente sea la reaparición de incontinencia. En el otro extremo del espectro de problemas clínicos, la elección de un manguito demasiado pequeño o de un reservorio con demasiada presión puede dar como resultado obstrucción infravesical ${ }^{9}$.

La técnica quirúrgica para la implantación del AMS-800 es relativamente simple pero un error en la elección manguito y el reservorio adecuados o durante la manipulación de las conexiones de los diferentes componentes puede dar como resultado el fracaso terapéutico. Muchos de los problemas derivados del AMS-800 son inherentes al propio diseño del esfinter ${ }^{9}$. Uno de ellos consiste en la imposibilidad de controlar la presión de oclusión, porque nunca se sabe si un reservorio de, por ejemplo, $61-70 \mathrm{~cm} . \mathrm{H}_{2} \mathrm{O}$ funciona en el limite de presión superior, medio o inferior. $\mathrm{Si}$ para mejorar la continencia se cambia por el de 71-80 cm. $\mathrm{H}_{2} \mathrm{O}$ la diferencia puede ser de $19 \mathrm{~cm}$. $\mathrm{H}_{2} \mathrm{O}$ (suficiente para producir erosión) o de tan sólo $1 \mathrm{~cm} . \mathrm{H}_{2} \mathrm{O}$ (el cambio de reservorio no sirvió para nada). Otro problema es que el manguito inflado del modelo AMS presenta la configuración de tres 'almohadas' por lo que la presión de oclusión no es homogénea, dando como resultado tres puntos de presión elevada y entre ellos zonas de baja presión, lo que favorece la erosión por un lado y la formación de irregularidades y grietas en la silicona, que predisponen al fallo mecánico, por otro. Por último, el AMS-800 ejerce una presión constante sobre la uretra o cuello vesical, independientemente de que los periodos en los que se necesitan presiones de oclusión tan elevadas sean puntuales, es decir, cuando el paciente tose o realiza esfuerzos.

Nuestros estudios en la fase pre-clínica del nuevo esfinter con la técnica de perfil de presión uretral con microtransductor multicanal simultánea con flujometría sanguínea mediante láserdoppler intrauretral ${ }^{10,11}$ en pacientes con el AMS800 demostraron que la continencia de los pacientes con esta prótesis depende de dos factores: 1) la presión que el manguito ejerce sobre la uretra bulbar y 2) la preservación de la perfusión sanguínea de la mucosa y submucosa uretral. Durante los periodos de reposo abdominal y para un determinado rango de presiones en el manguito, el factor más importante es la perfusión sanguínea uretral: los pacientes con buena perfusión permanecerán continentes y aquellos en los que la isquemia impide el efecto de sellado de la mucosa y submucosa desarrollarán incontinencia. Además se estableció que las presiones de oclusión superiores a $40 \mathrm{~cm}$ de $\mathrm{H}_{2} \mathrm{O}$ reducen significativamente la irrigación uretral predisponiendo a la atrofia y erosión.

El esfinter artificial FlowSecure incorpora una serie de características innovadoras para resolver los problemas derivados del diseño del AMS-800, facilitar la técnica quirúrgica, disminuir el número de reintervenciones y conseguir una mejor tasa de continencia en los pacientes. Los resultados a relativamente corto plazo ${ }^{12}$ del modelo FlowSecure parecen conseguir todos estos objetivos para los que fue creado y son alentadores. La técnica quirúrgica es simple, rápida, fácilmente reproducible y no requiere la interconexión de los componentes por lo que se evitan posibles errores de manipulación o entrada de burbujas de aire y partículas de sangre o grasa en el sistema. El sistema se implanta a presión atmosférica, minimizando los riesgos de isquemia postoperatoria hasta que los tejidos estén revascularizados. Durante la presurización del sistema, el reservorio regulador de presión del FlowSecure se establece a la mínima presión necesaria para producir continencia en reposo que no suele superar los $40 \mathrm{~cm} . \mathrm{H}_{2} \mathrm{O}$ en el interior de la uretra, mientras que el reservorio de menor presión del AMS-800 supera constantemente $\operatorname{los} 40 \mathrm{~cm} . \mathrm{H}_{2} \mathrm{O}$ para mantener la continencia. El reservorio de asistencia al estrés sólo eleva la presión de oclusión del manguito cuando aumenta la presión intra-abdominal, volviendo a sus valores basales cuando el esfuerzo desaparece. Gracias al diseño del manguito en forma de cinturón se permite la adaptación individualizada a cada uretra, se minimizan los riesgos de atrofia y erosión y se evita la formación de grietas que podrían provocar perforaciones en la silicona. Gracias al puer- 
to autosellable de la bomba de control, el sistema se puede regular dependiendo de las necesidades clínicas de cada paciente sin necesidad de reintervenciones. En aquellos pacientes en los que persista o reaparezca la incontinencia se puede inyectar mayor cantidad de volumen hasta que se restaure la continencia. En los pacientes en los que se pueda producir dificultad miccional por excesiva presión de oclusión se puede extraer líquido través del mismo puerto.

A pesar que el nuevo esfinter FlowSecure constituye un diseño dinámico que podría evitar las complicaciones del AMS-800 y proporcionar una mejor tasa de continencia, todas las técnicas quirúrgicas para el tratamiento de la incontinencia de esfuerzo han sufrido un proceso histórico común: ensayo, error, nuevo ensayo con grandes esperanzas, desesperación con los resultados a largo plazo y, finalmente, búsqueda de un tratamiento diferente por fracaso de la técnica. Por eso existen tantas técnicas diferentes para el tratamiento de la incontinencia de esfuerzo. Habrá que esperar unos años para obtener resultados a largo plazo y comprobar si el FlowSecure se convierte en el esfinter artificial ideal para el tratamiento de la incontinencia de esfuerzo refractaria a otros procedimientos.

\section{CONCLUSIONES}

El esfinter urinario artificial FlowSecure constituye un nuevo concepto de esfinter artificial dinámico para el tratamiento de la incontinencia de esfuerzo. La introducción de un reservorio de asistencia al estrés con oclusión condicional permite establecer presiones de oclusión basal bajas preservando la irrigación uretral y por tanto reduciendo el riesgo de complicaciones. Además de ser fácilmente implantable, permite modificar la presión del sistema sin necesidad de intervenciones mediante la inyección o extracción de líquido del sistema. Las innovaciones en el manguito no sólo permiten una mejor adaptación a la uretra si no que reducen la posibilidad de daño de la silicona y por tanto fallo mecánico. Habrá que esperar a obtener resultados a más largo plazo para establecer el lugar que esta prótesis puede llegar a ocupar en el tratamiento de la incontinencia de esfuerzo, aunque los resultados preliminares son muy alentadores.

\section{REFERENCIAS}

1. Foley FEB. An artificial sphincter: A new device and operation for control of enuresis and urinary incontinence. General considerations, indications and results. J Urol. 1947;58:250-259.

2. Scott FB, Bradley WE, Timm GW. Treatment of urinary incontinence by implantable prosthetic sphincter. Urology 1973;1(3):252-259.

3. Hajivassiliou CA. A review of the complications and results of implantation of the AMS artificial urinary sphincter. Eur Urol. 1999;35(1)36-44.

4. Kreder KJ, Webster GD. Evaluation and management of incontinence after implantation of the Artificial Urinary Sphincter. Urol Clin N Amer. 1991; 18(2):375-381.

5. Mundy AR. Artificial Sphincters. Br J Urol. 1991; 67(3):225-229.

6. Nurse DA and Mundy AR. One hundred artificial sphincters. Br J Urol. 1988;61(4):318-325.

7. Craggs MD. An adjustable pressure-regulated prosthetic sphincter. J Physiol. 1986;377,6P.

8. Craggs MD, Chaffey NJ, Mundy AR. A preliminary report on a new hydraulic sphincter for controlling urinary incontinence. J Med Eng and Tech. 1991; 15(2):58-62.

9. Garcia Montes F, Mundy AR. Artificial Urinary Sphincters: How do they work? Why do they fail?. Continuing Medical Education in Urology 1999;3: 7-9.

10. Garcia Montes F, Knight S, Mundy AR, Craggs MD. The significance of low urethral blood flow in recurrent incontinence in patients with a long standing artificial urinary sphincter. Neurourol and Urodyn. 1999;18(4):394-396.

11. Garcia Montes F, Knight S, Mundy AR, Craggs MD. Effects of artificial urinary sphincters on urethral blood perfusion measured with laser Doppler. Br J Urol - Int. 1999;8(1):154-155.

12. Knight S, Susser J, Greenwell T, Mundy AR, Craggs MD. A new artificial sphincter with conditional occlusion for stress urinary incontinence: Preliminary clinical results. European Urology 2006;50 (3):574-580.

Correspondencia autor: Dr. F. García Montes

Servicio de Urología. Hospital Universitario Son Dureta Andrea Doria, 55. 07014 Palma de Mallorca. Baleares. Tel.: 971175000

E-mail autor: fgarciamontes@gmail.com

Información artículo: Original - Incontinencia masculina

Trabajo recibido: diciembre 2006

Trabajo aceptado: febrero 2007 